\title{
Vertical distribution of particle-phase dicarboxylic acids, oxoacids and $\alpha$-dicarbonyls in the urban boundary layer based on the 325-meter tower in Beijing
}

5 Wanyu Zhao ${ }^{1,5,6}$, Hong Ren², Kimitaka Kawamura ${ }^{3,5}$, Huiyun Du ${ }^{1,6}$, Xueshun Chen ${ }^{1,6}$, Siyao Yue ${ }^{1,6}$, Qiaorong Xie ${ }^{1,6}$, Lianfang Wei ${ }^{1,6}$, Ping Li ${ }^{1,6}$, Xin Zeng ${ }^{4}$, Shaofei Kong ${ }^{4}$, Yele Sun ${ }^{1}$, Zifa Wang ${ }^{1}$, and Pingqing $\mathrm{Fu}^{2 *}$

${ }^{1}$ State Key Laboratory of Atmospheric Boundary Layer Physics and Atmospheric Chemistry, Institute of Atmospheric Physics, Chinese Academy of Sciences, Beijing 100029, China

$10{ }^{2}$ Institute of Surface-Earth System Science, Tianjin University, Tianjin 300072, China

${ }^{3}$ Chubu Institute for Advanced Studies, Chubu University, Kasugai 487-8501, Japan

${ }^{4}$ Department of Atmospheric Science, School of Environmental Sciences, China University of Geosciences, Wuhan, 430074, China ${ }^{5}$ Institute of Low Temperature Science, Hokkaido University, Sapporo 060-0819, Japan

${ }^{6}$ College of Earth and Planetary Sciences, University of Chinese Academy of Sciences, Beijing 100049, China

15 Correspondence to: Pingqing Fu (fupingqing@tju.edu.cn) 
Table S1. Average vertical ratios of diacids and related organic compounds observed at the tower in Beijing in summer 2015.

\begin{tabular}{|l|c|c|c|c|c|c|}
\hline Ratio & Ground level $(8 \mathrm{~m})$ & Mean $\pm \mathrm{SD}$ & $120 \mathrm{~m}$ & Mean $\pm \mathrm{SD}$ & $260 \mathrm{~m}$ & Mean $\pm \mathrm{SD}$ \\
\hline OC/EC & $5.0-10$ & $7.5 \pm 1.5$ & $2.6-12$ & $7.0 \pm 2.2$ & $2.5-12$ & $7.0 \pm 2.4$ \\
\hline SOC/POC & $\mathrm{NA}-1.2$ & $0.5 \pm 0.3$ & NA-3.6 & $1.8 \pm 0.8$ & NA-3.6 & $1.9 \pm 0.9$ \\
\hline $\mathrm{C}_{3} / \mathrm{C}_{4}$ & $0.77-1.3$ & $1.1 \pm 0.14$ & $0.6-1.4$ & $1.1 \pm 0.2$ & $0.65-1.4$ & $1.2 \pm 0.21$ \\
\hline C9/total diacids & $0.04-0.13$ & $0.07 \pm 0.02$ & $0.03-0.13$ & $0.06 \pm 0.02$ & $0.03-0.07$ & $0.05 \pm 0.01$ \\
\hline diacids-C/OC & $0.9-2.2$ & $1.5 \pm 0.3$ & $1.1-3.0$ & $1.6 \pm 0.4$ & $1.2-2.5$ & $1.7 \pm 0.3$ \\
\hline diacids-C/TC & $0.8-2.0$ & $1.4 \pm 0.3$ & $0.9-2.1$ & $1.4 \pm 0.3$ & $1.2-1.9$ & $1.5 \pm 0.3$ \\
\hline oxoacids-C/OC & $0.75-0.35$ & $0.23 \pm 0.06$ & $0.03-0.66$ & $0.27 \pm 0.11$ & $0.14-0.4$ & $0.27 \pm 0.06$ \\
\hline oxoacids-C/TC & $0.06-0.31$ & $0.21 \pm 0.05$ & $0.02-0.47$ & $0.23 \pm 0.09$ & $0.12-0.35$ & $0.23 \pm 0.05$ \\
\hline dicarbonyls-C/OC & $0.03-0.09$ & $0.04 \pm 0.02$ & $0.0-0.11$ & $0.05 \pm 0.03$ & $0.03-0.1$ & $0.06 \pm 0.03$ \\
\hline dicarbonyls-C/TC & $0.02-0.08$ & $0.04 \pm 0.01$ & $0.0-0.1$ & $0.04 \pm 0.02$ & $0.03-0.1$ & $0.05 \pm 0.02$ \\
\hline
\end{tabular}

$\mathrm{NA}=$ not available . 
Table S2. The decrease percent of diacids and related compound concentrations in restriction period compared to the first (DR/N1, \%) and second (DR/N2, \%) non-restriction period in Beijing.

\begin{tabular}{|c|c|c|c|c|c|c|}
\hline & \multicolumn{3}{|c|}{ DR/N1 } & \multicolumn{3}{|c|}{$\mathrm{DR} / \mathrm{N} 2$} \\
\hline & $\begin{array}{c}\text { Ground } \\
\text { level }(8 \mathrm{~m})\end{array}$ & $120 \mathrm{~m}$ & $260 \mathrm{~m}$ & $\begin{array}{c}\text { Ground } \\
\text { level }(8 \mathrm{~m})\end{array}$ & $120 \mathrm{~m}$ & $260 \mathrm{~m}$ \\
\hline $\mathrm{C}_{2}$ & 51 & 57 & 59 & 26 & 29 & 34 \\
\hline $\mathrm{C}_{3}$ & 44 & 50 & 51 & 23 & 18 & 25 \\
\hline $\mathrm{C}_{4}$ & 39 & 52 & 53 & 25 & 29 & 33 \\
\hline $\mathrm{C}_{5}$ & 43 & 50 & 50 & 24 & 30 & 36 \\
\hline $\mathrm{C}_{6}$ & 43 & 39 & ND & 14 & 16 & ND \\
\hline $\mathrm{C}_{9}$ & 18 & 29 & 41 & 27 & 20 & 18 \\
\hline $\mathrm{Ph}$ & 27 & 41 & 41 & ND & 10 & 14 \\
\hline $\mathrm{tPh}$ & 63 & 67 & 69 & 42 & 46 & 55 \\
\hline Pyr & 55 & 64 & 66 & 40 & 52 & 42 \\
\hline$\omega \mathrm{C}_{2}$ & 63 & 59 & 65 & 40 & 41 & 45 \\
\hline Gly & 44 & 45 & 55 & 31 & 40 & 38 \\
\hline MeGly & 53 & 41 & 55 & 27 & 37 & 39 \\
\hline Total diacids & 47 & 53 & 55 & 22 & 27 & 31 \\
\hline Total oxoacids & 52 & 53 & 58 & 31 & 32 & 36 \\
\hline Total dicarbonyls & 50 & 43 & 55 & 29 & 36 & 39 \\
\hline
\end{tabular}

$\mathrm{ND}=$ no decrease. 
Table S3. The P/T ratios of $\mathrm{C}_{2}-\mathrm{C} / \mathrm{WSOC}$, Pyr-C/WSOC, $\omega_{2}-\mathrm{C} / \mathrm{WSOC}$, Gly-C/WSOC and MeGly-C/WSOC observed at the tower in Beijing in summer 2015.

\begin{tabular}{|l|c|c|c|c|c|c|}
\hline & \multicolumn{3}{|c|}{ P1/T1 } & \multicolumn{3}{c|}{ P3/T3 } \\
\hline & $\begin{array}{c}\text { Ground } \\
\text { level (8 m) }\end{array}$ & $120 \mathrm{~m}$ & $260 \mathrm{~m}$ & $\begin{array}{c}\text { Ground } \\
\text { level (8 m) }\end{array}$ & $120 \mathrm{~m}$ & $260 \mathrm{~m}$ \\
\hline $\mathrm{C}_{2}$-C/WSOC & 2.3 & 1.0 & 1.3 & 4.1 & 1.4 & 1.2 \\
\hline Pyr-C/WSOC & 2.7 & 0.9 & 1.8 & 5.6 & 1.5 & 1.5 \\
\hline$\omega C_{2}$-C/WSOC & 2.3 & 1.3 & 1.5 & 5.2 & 1.8 & 1.4 \\
\hline Gly-C/WSOC & 2.0 & 1.3 & 1.4 & 5.7 & 1.8 & 1.6 \\
\hline MeGly-C/WSOC & 1.4 & 0.9 & 1.8 & 5.8 & 2.0 & 1.5 \\
\hline
\end{tabular}


Table S4. Summary of error estimation diagnostics from BS and DISP for PMF.

\begin{tabular}{|c|c|c|c|c|c|c|c|c|}
\hline \multicolumn{2}{|c|}{$\begin{array}{c}\text { BS Mapping } \\
\quad(\mathrm{R} \geq 0.6)\end{array}$} & $\begin{array}{l}\text { Secondary } \\
\text { sulfate } \\
\text { formation }\end{array}$ & $\begin{array}{c}\text { Secondary } \\
\text { nitrate } \\
\text { formation }\end{array}$ & $\begin{array}{l}\text { Plants } \\
\text { emissions }\end{array}$ & $\begin{array}{l}\text { Biomass } \\
\text { burning }\end{array}$ & $\begin{array}{l}\text { Vehicle } \\
\text { exhausts }\end{array}$ & $\begin{array}{c}\text { Coal } \\
\text { combustion }\end{array}$ & Unmapped \\
\hline \multicolumn{2}{|c|}{$\begin{array}{c}\text { Secondary sulfate } \\
\text { formation }\end{array}$} & 49 & 0 & 1 & 0 & 0 & 0 & 0 \\
\hline \multicolumn{2}{|c|}{$\begin{array}{c}\text { Secondary nitrate } \\
\text { formation }\end{array}$} & 0 & 50 & 1.8 & 0 & 0 & 0 & 0 \\
\hline \multicolumn{2}{|c|}{ Plants emissions } & 8 & 0 & 41 & 0 & 0 & 1 & 0 \\
\hline \multicolumn{2}{|c|}{ Biomass burning } & 0 & 0 & 0 & 50 & 0 & 0 & 0 \\
\hline \multicolumn{2}{|c|}{ Vehicle exhausts } & 0 & 0 & 0 & 0 & 50 & 0 & 0 \\
\hline \multicolumn{2}{|c|}{ Coal combustion } & 0 & 0 & 0 & 0 & 0 & 50 & 0 \\
\hline \multicolumn{2}{|c|}{ DISP Diagnostics } & \multicolumn{3}{|c|}{ Error code: 0} & \multicolumn{3}{|c|}{ Largest decrease in $Q:-0.031$} & \\
\hline \multirow{4}{*}{$\begin{array}{l}\text { Factor } \\
\text { Swaps }\end{array}$} & $\mathrm{d} Q^{\max }=4$ & 0 & 0 & 0 & 0 & 0 & 0 & \\
\hline & $\mathrm{d} Q^{\max =8}$ & 4 & 0 & 4 & 0 & 0 & 4 & \\
\hline & $\mathrm{d} Q^{\max }=15$ & 12 & 0 & 12 & 0 & 0 & 9 & \\
\hline & $\mathrm{d} Q^{\max }=25$ & 18 & 0 & 18 & 1 & 0 & 11 & \\
\hline
\end{tabular}


(a)
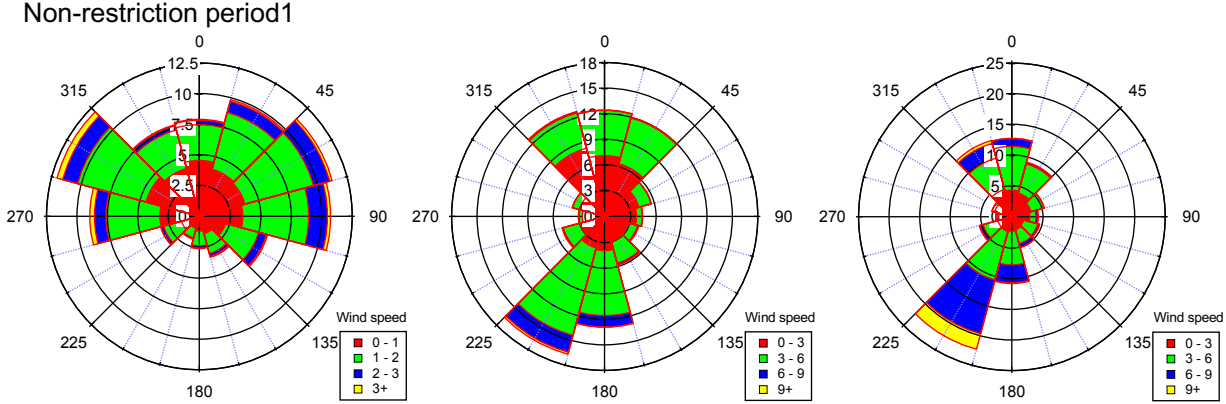

Restriction period
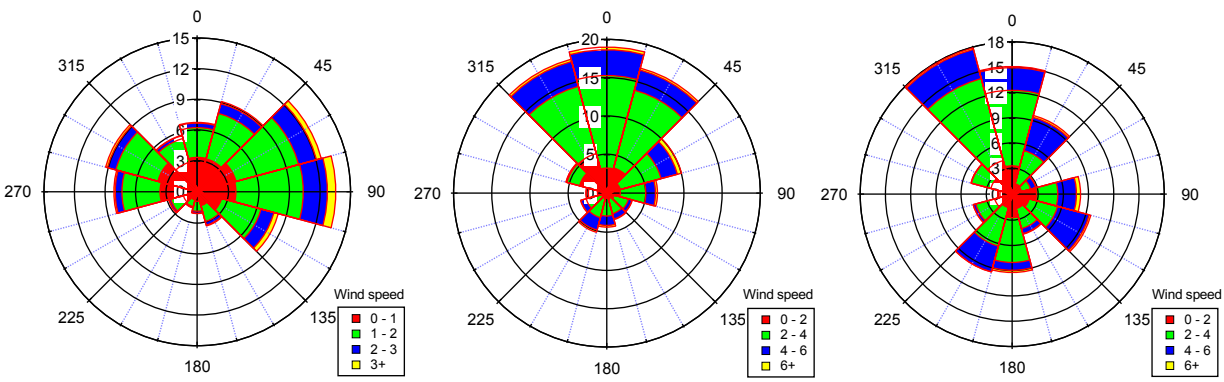

Non-restriction period2
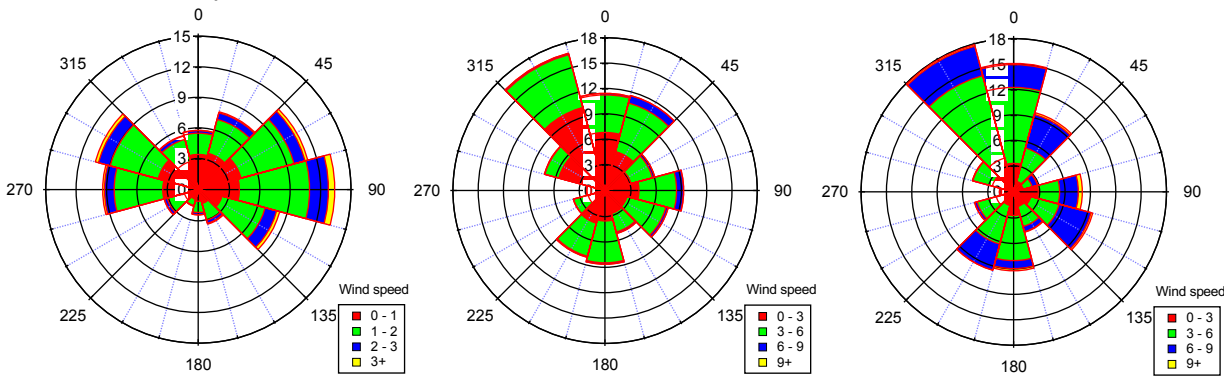

(b)

$8 m$

$120 \mathrm{~m}$

$280 \mathrm{~m}$

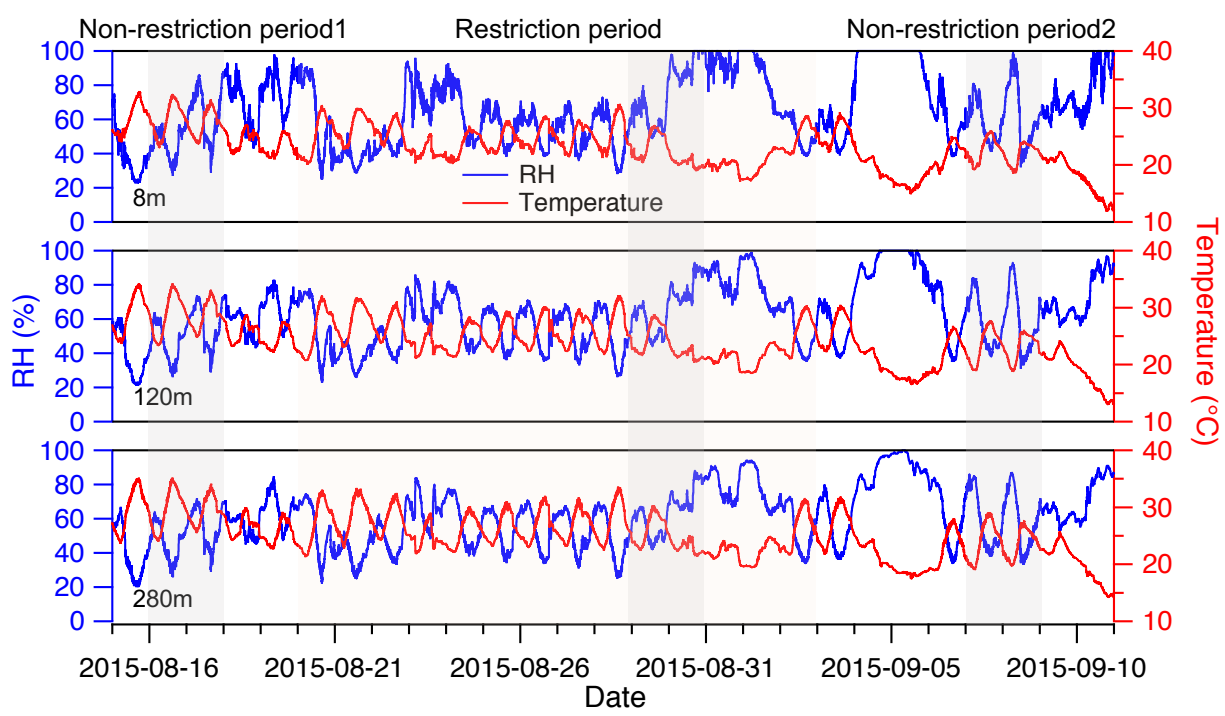

Figure S1: (a) Wind rose diagrams, (b) time series of temperature $\left({ }^{\circ} \mathrm{C}\right)$ and relative humidity $(\%)$ at ground level, $120 \mathrm{~m}$ and $280 \mathrm{~m}$ in Beijing in summer 2015. 

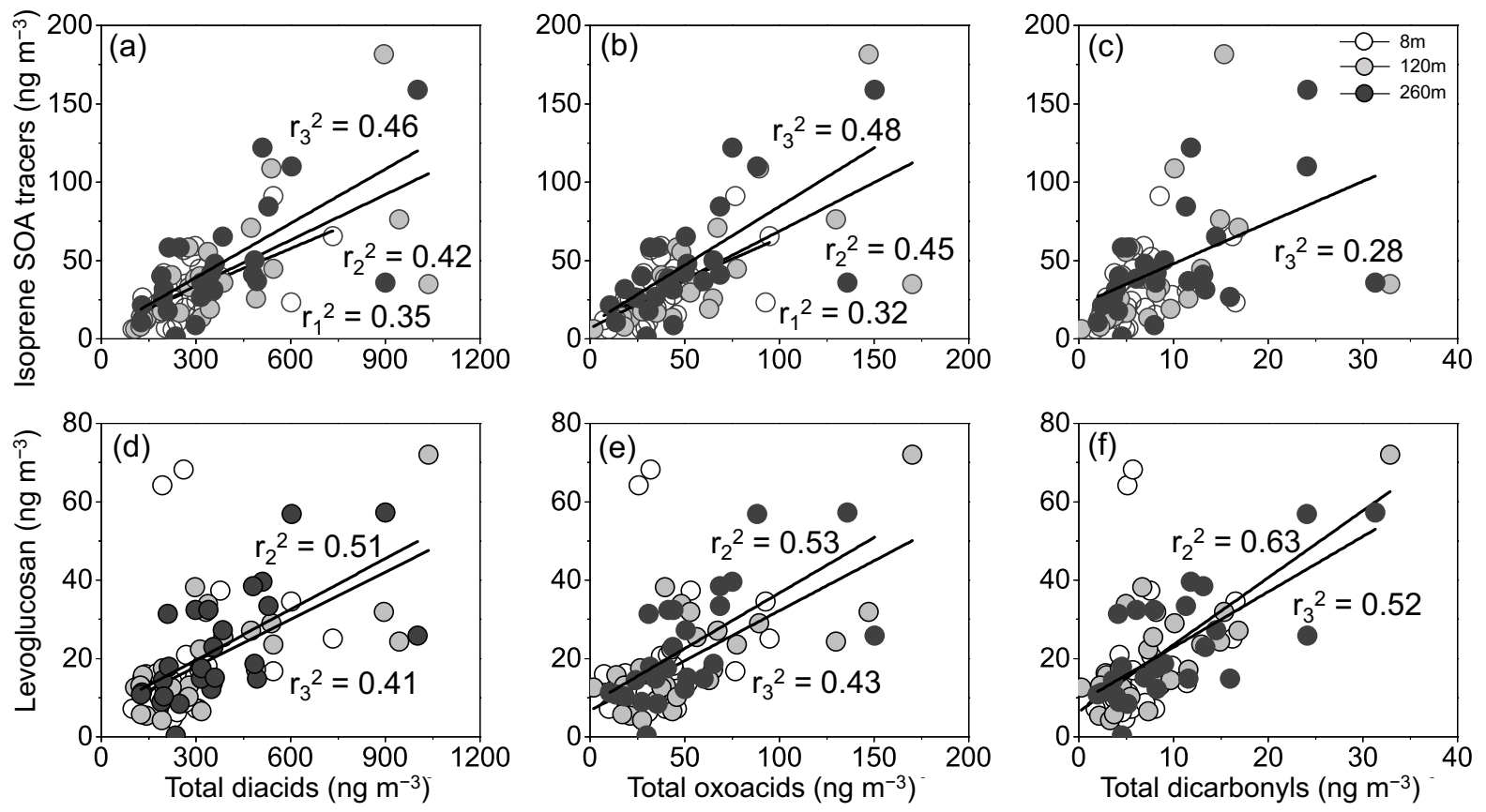

Figure S2: Linear relationships for total diacids, oxoacids and dicarbonyls with levoglucosan, isoprene SOA tracers at ground level, 120 $\mathrm{m}$ and $260 \mathrm{~m}$ in Beijing. The $r_{1}^{2}, r_{2}^{2}$, and $r_{3}^{2}$ represent the correlation coefficients at $8 \mathrm{~m}, 120 \mathrm{~m}$ and $260 \mathrm{~m}$, respectively. 


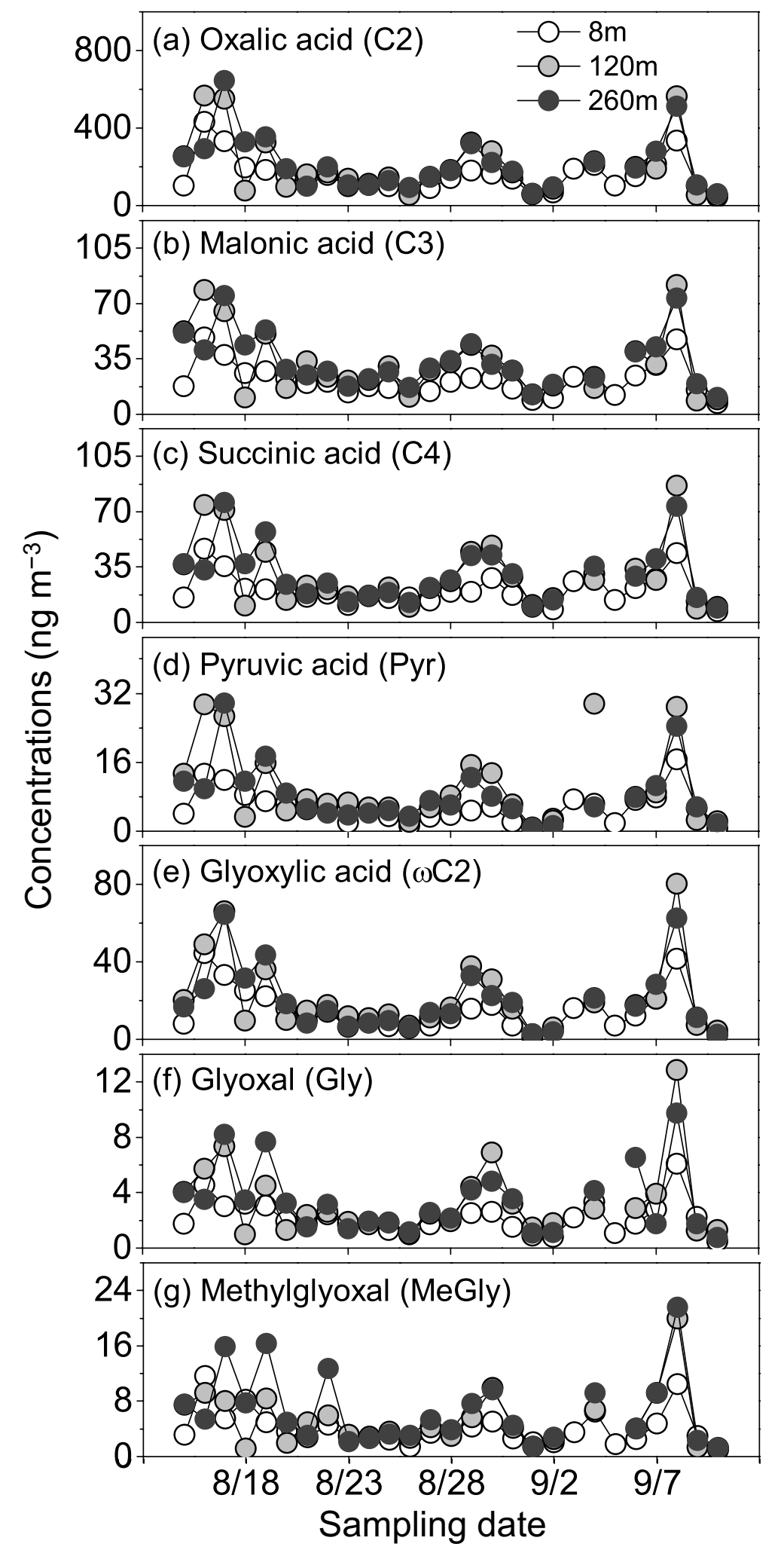

Figure S3: Daily variations in the concentrations of selected organic acids at three sampling layers in Beijing. 

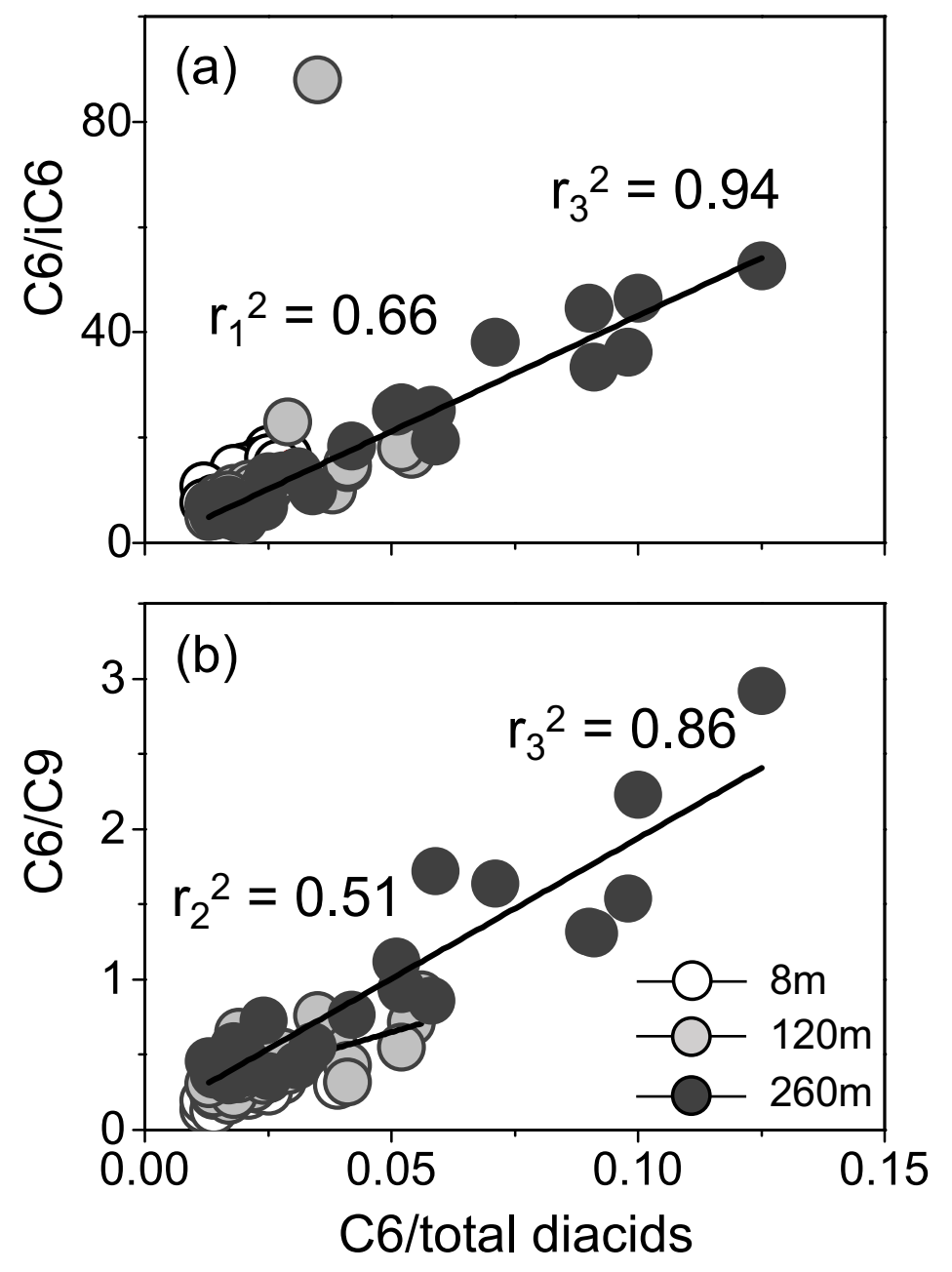

Figure S4: Relationships for $C_{6} /$ total diacids with $C_{6} / C_{6}$ and $C_{6} / C_{9}$ in Beijing in summer 2015. The $r_{1}^{2}, r_{2}^{2}$, and $r_{3}^{2}$ represent the correlation coefficients at $8 \mathrm{~m}, 120 \mathrm{~m}$ and $260 \mathrm{~m}$, respectively. 


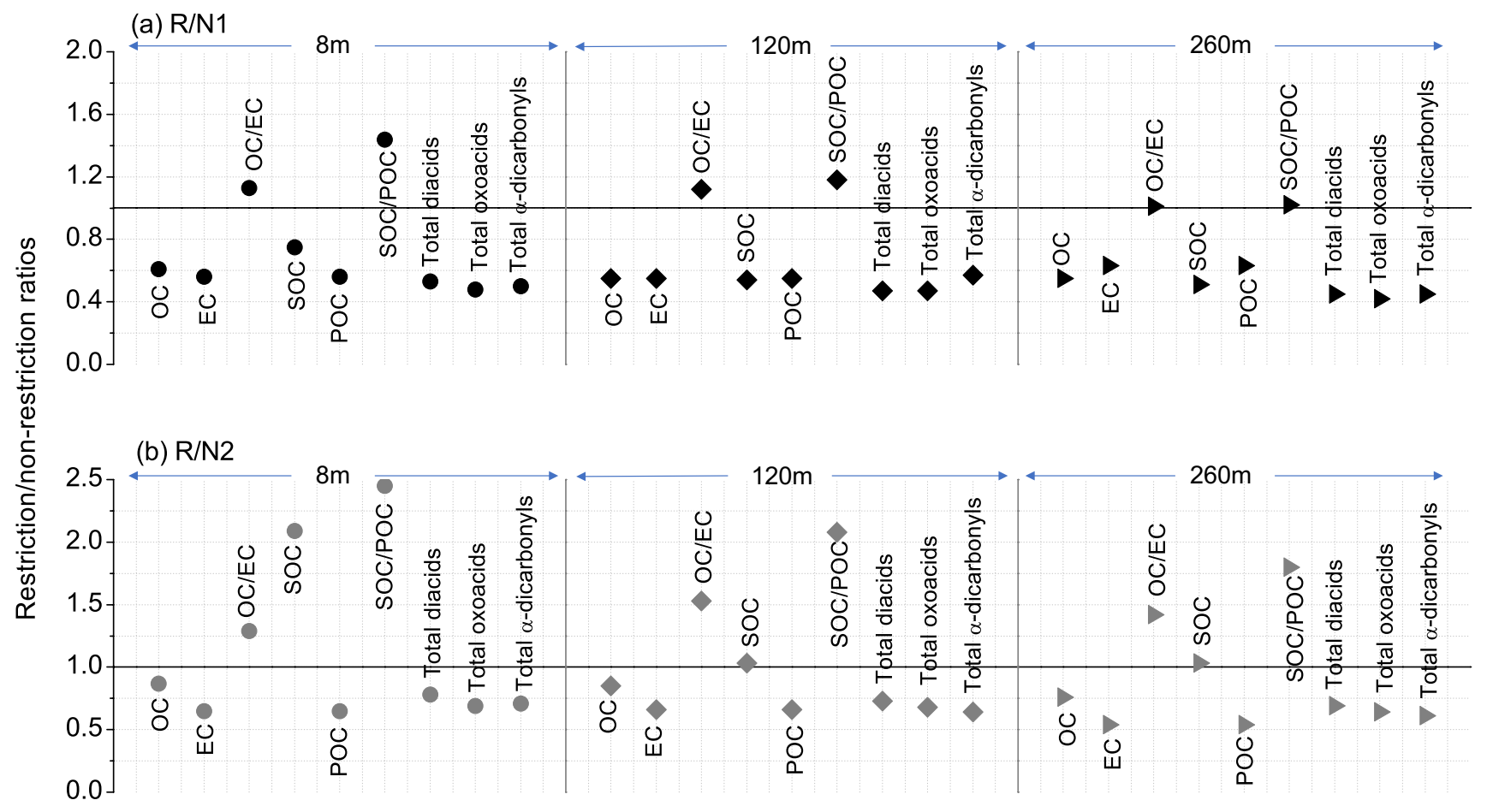

Figure S5: The R/N ratios of particulate compounds observed at the tower in Beijing in summer 2015. 


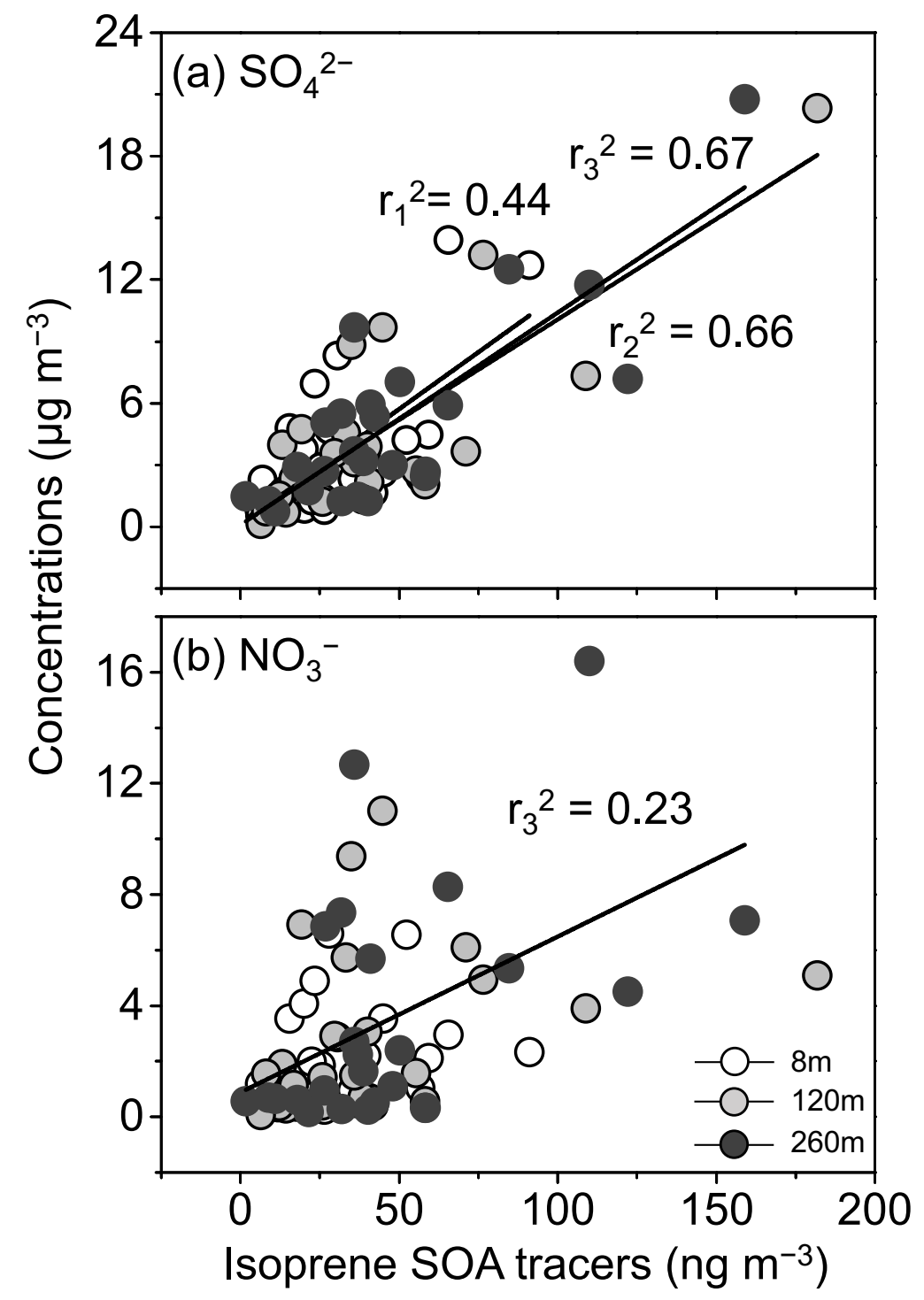

Figure S6: Relationships for isoprene SOA tracers with $\mathrm{SO}_{4}{ }^{2-}$ and $\mathrm{NO}_{3}{ }^{-}$at ground level, $120 \mathrm{~m}$ and $260 \mathrm{~m}$. The $\mathbf{r}_{1}{ }^{2}, \mathrm{r}_{2}{ }^{2}$, and $\mathrm{r}_{3}{ }^{2}$ represent the correlation coefficients at $8 \mathrm{~m}, 120 \mathrm{~m}$ and $260 \mathrm{~m}$, respectively. 